\title{
Pontiac Fever
}

National Cancer Institute

\section{Source}

National Cancer Institute. Pontiac Fever. NCI Thesaurus. Code C128335.

A self-limited, febrile illness without pneumonia that occurs in epidemics, and that is caused by Legionella species. 